Supporting Information

$$
\text { of }
$$

\title{
Platelet-Mimicking Biotaxis Targeting Vasculature-Disrupted Tumors for Cascade Amplification of Hypoxia-Sensitive Therapy
}

Mingkang Zhang ${ }^{\S}$, Jing-Jie Ye ${ }^{\S}$, Yu Xia, Zi-Yang Wang, Chu-Xin Li, Xiao-Shuang Wang, Wuyang Yu, Wen Song, Jun Feng* and Xian-Zheng Zhang

Key Laboratory of Biomedical Polymers of Ministry of Education \& Department of Chemistry, Wuhan University, Wuhan 430072, P.R. China

* Corresponding author. E-mail: fengjun@whu.edu.cn

$\S$ M. Zhang and J.-J. Ye contributed equally to this work. 
Table of Contents for Supporting Information:

Figure S1. SEM image of amino modified MSN nanoparticles.....................5

Figure S2. Barrett-Joyner-Halenda (BJH) pore size distribution of amino modified

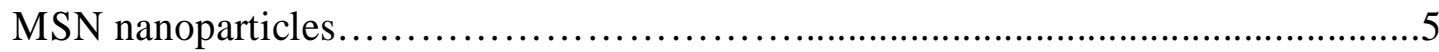

Figure S3. Zeta potential of amino modified MSN, MT, MTD and MTD@P nanoparticles, respectively .....................................................

Figure S4. Standard absorbance-concentration curves of DMXAA (245 nm) and TPZ

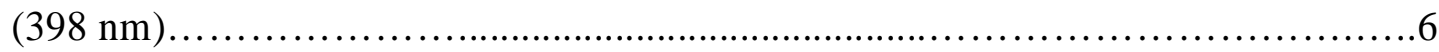

Figure S5. (A) FACS study of purified platelets that were doubly stained with FITCconjugated, anti-CD45 (white blood cell marker) and anti-CD41 (platelet marker). (B) Bright and fluorescence CLSM images of the collected platelet labeled with CD41-

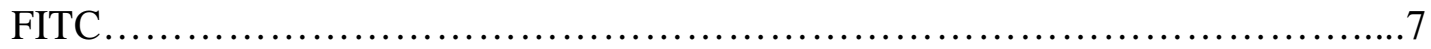

Figure S6. Hydrodynamic diameters of MTD and MTD@P nanoparticles............7

Figure S7. The stability test of MTD@P nanoparticles in different medium for 7 days. 8

Figure S8. The in vitro release profile of TPZ (A) and DMXAA (B) from TPZ or DMXAA, MTD and MTD@P at pH 7.4...........................................

Figure S9. SDS-PAGE protein analysis of MSN, MTD@P and PM...........................9

Figure S10. (A) Quantitative analysis toward the fluorescence intensity in MAEC cells after 4 h co-incubation with $100 \mu \mathrm{g} / \mathrm{mL}$ of MTD@P, MTD@R, MTD and MTD@P (+ represented the cells were pre-treated with antibody pretreated) (B) Mean fluorescence intensity in MAEC cells after different treatments..............................9 
Figure S11. (A) Fluorescence images of RAW264.7 cells after the treatments with MTD and MTD@P, respectively. (B) Quantitative analysis toward the fluorescence intensity in RAW264.7 cells after 2 h co-incubation with MTD and MTD@P. (C) Mean fluorescence intensity of DIR labeled nanoparticles in RAW264.7 cells after different

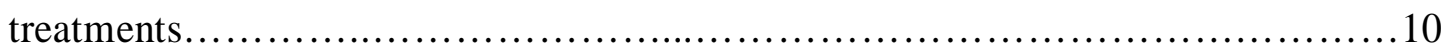

Figure S12. Hemolysis test of MTD@P at different concentrations (0, 50, 100, 200, $400 \mu \mathrm{g} / \mathrm{mL})$ in PBS .10

Figure S13. Western blotting analysis for P-Selectin expression on PM and MTD@P...... .11

Figure S14. (A) Quantitative analysis toward the fluorescence intensity in 3T3 and CT26 cell after 2 h co-incubation with $100 \mu \mathrm{g} / \mathrm{mL}$ of MTD@P. (B) Mean fluorescence intensity in 3T3 and CT26 cell after co-incubation with MTD@P

Figure S15. Fluorescence images of 3T3 (upper) and CT26 (lower) cells after 2 h co-

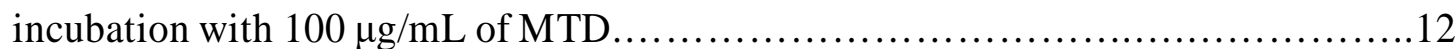
Figure S16. (A) Variation of mean fluorescence intensity at tumor sites in the mice with time after the treatments with MTD@P, MT@P, MTD@R and MTD. (B) Mean fluorescence intensity of major organs in the mice treated with MTD@P, MT@P, MTD@R and MTD. (He: Heart; Li: Liver; Sp: Spleen; Lu: Lung; Ki: Kidney; Tu: Tumor). The organs were obtained at $72 \mathrm{~h}$ post injection 12

Figure S17. In vivo pharmacokinetic curves of TPZ (A) and DMXAA (B) after intravenous injection of free TPZ or DMXAA and MTD@P into mice, respectively...13 Figure S18. Variation of intratumoral average $\mathrm{sO}_{2}$ in different groups obtained before 
(0 h) and after (4 h) the administrations with different nanoparticles.

Figure S19. Mean fluorescence intensity in immunofluorescence images of the tumor slices stained with of pimonidazole antibody after different treatments. .14

Figure S20. Mean fluorescence intensity in immunofluorescence images of the tumor slices stained with of HIF-1 $\alpha$ antibody after different treatments. .14

Figure S21. Mean fluorescence intensity of TUNEL (A) and caspase-3 (B) antibody staining images of the tumor slices after different treatments .15

Figure S22. H\&E staining images of major organ slices after different treatments...15 Figure S23. Full scan H\&E staining images of major organ slices of healthy mice. Scale bar: $1 \mathrm{~mm}$ .16

Figure S24. Full scan H\&E staining images of major organ slices of CT26 tumorbearing mice. Scale bar: $1 \mathrm{~mm}$. Bleeding spots are indicated by black arrows.

Table S1 Blood routine analysis post different treatments (Major items: RBC: red blood cell, WBC: white blood cell, PLT: platelets, HCT: hematocrit, MCV: mean corpuscular volume, HGB: hemoglobin). .18

Table S2 Blood biochemistry analysis post different treatments. (TP: total protein, ALB: albumin, GLO: globulin, TBIL: total bilirubin, ALT: alanine aminotransferase, AST: aspartate aminotransferase, GGT: $\gamma$-glutamyl transpeptidase, UREA: urea, GLU: glucose). .18 


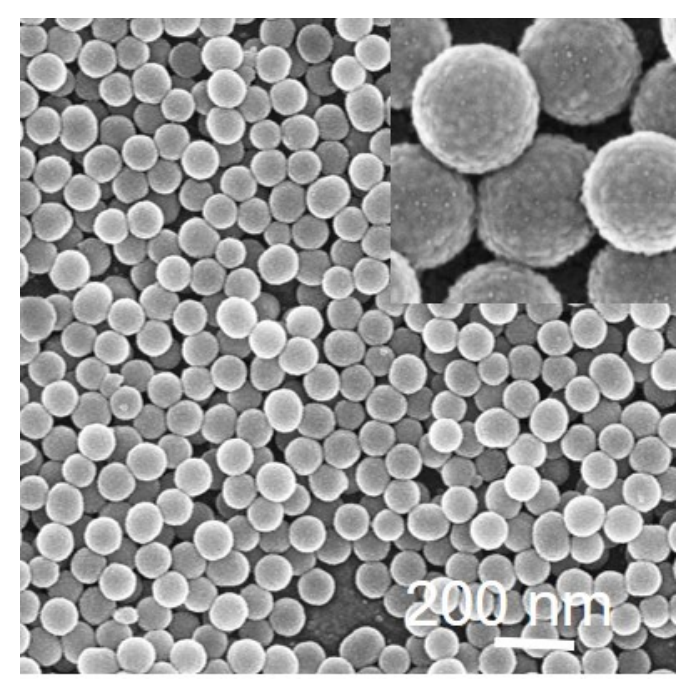

Figure S1. SEM image of amino modified MSN nanoparticles.

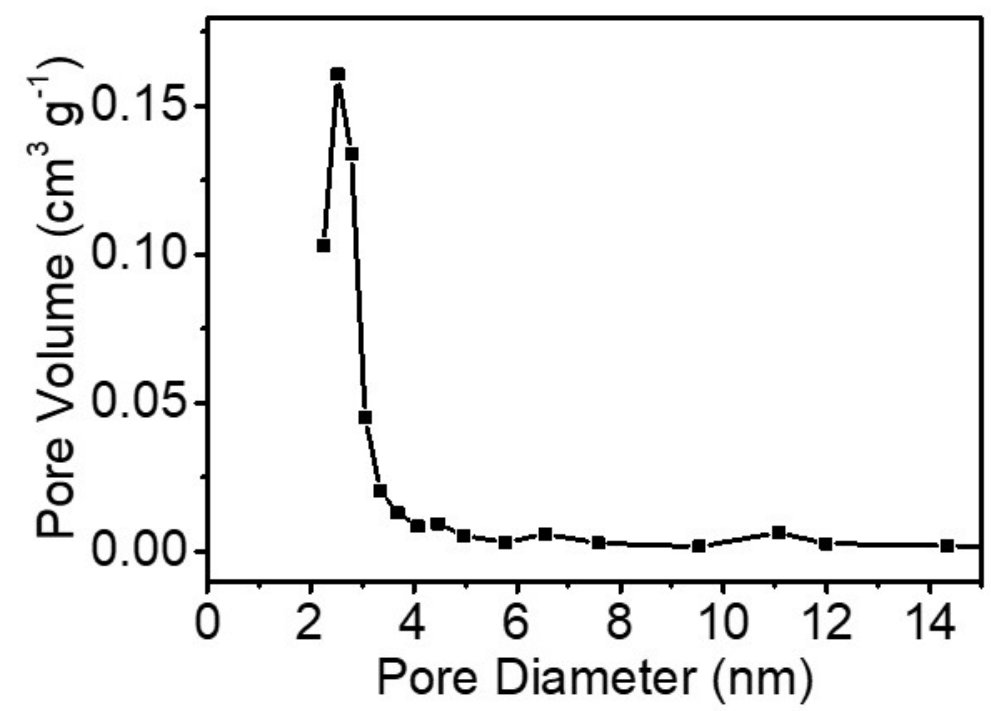

Figure S2. Barrett-Joyner-Halenda (BJH) pore size distribution of amino modified MSN nanoparticles. 


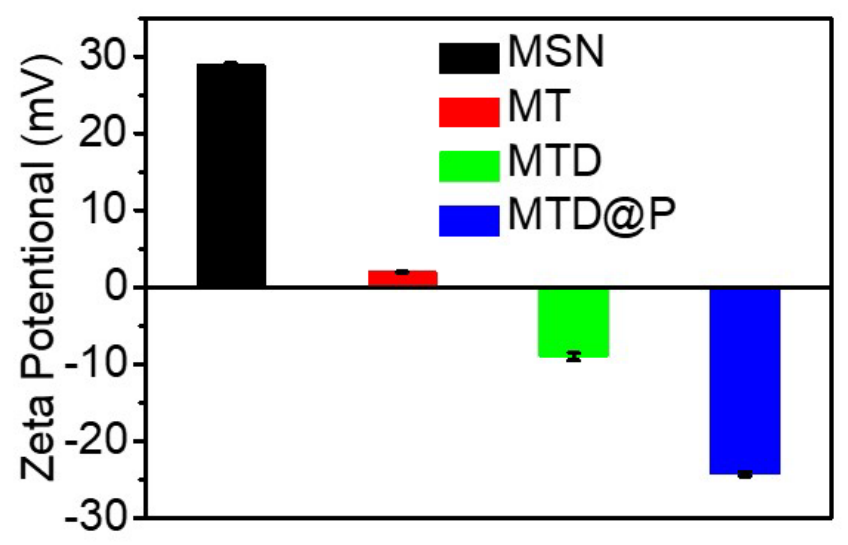

Figure S3. Zeta potential of amino modified MSN, MT, MTD and MTD@P nanoparticles, respectively.
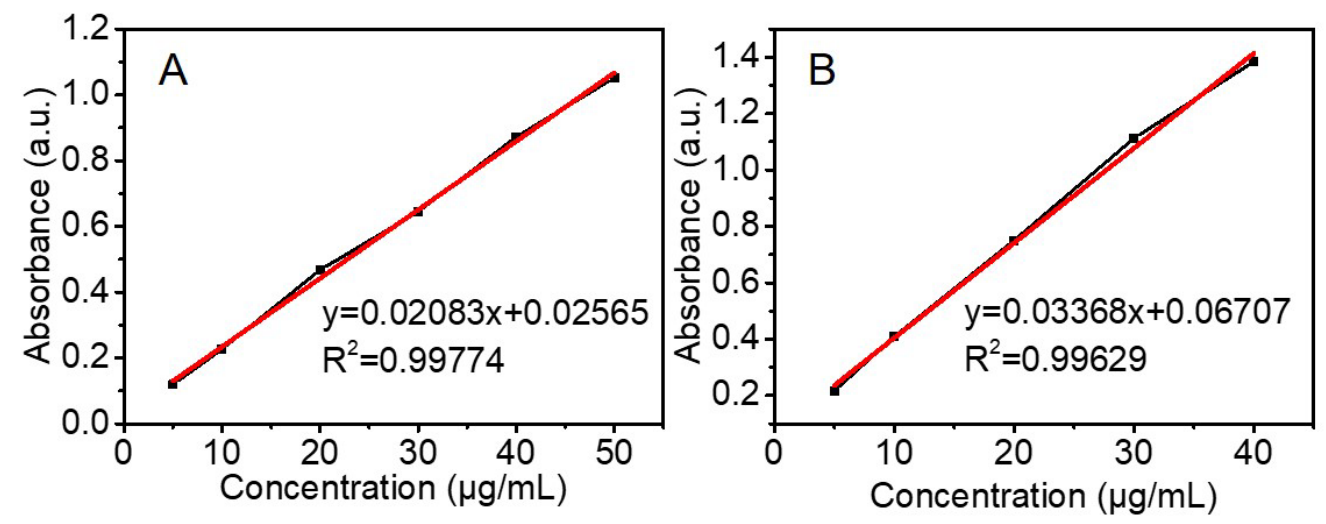

Figure S4. Standard absorbance-concentration curves of DMXAA (245 nm) and TPZ (398 nm). 

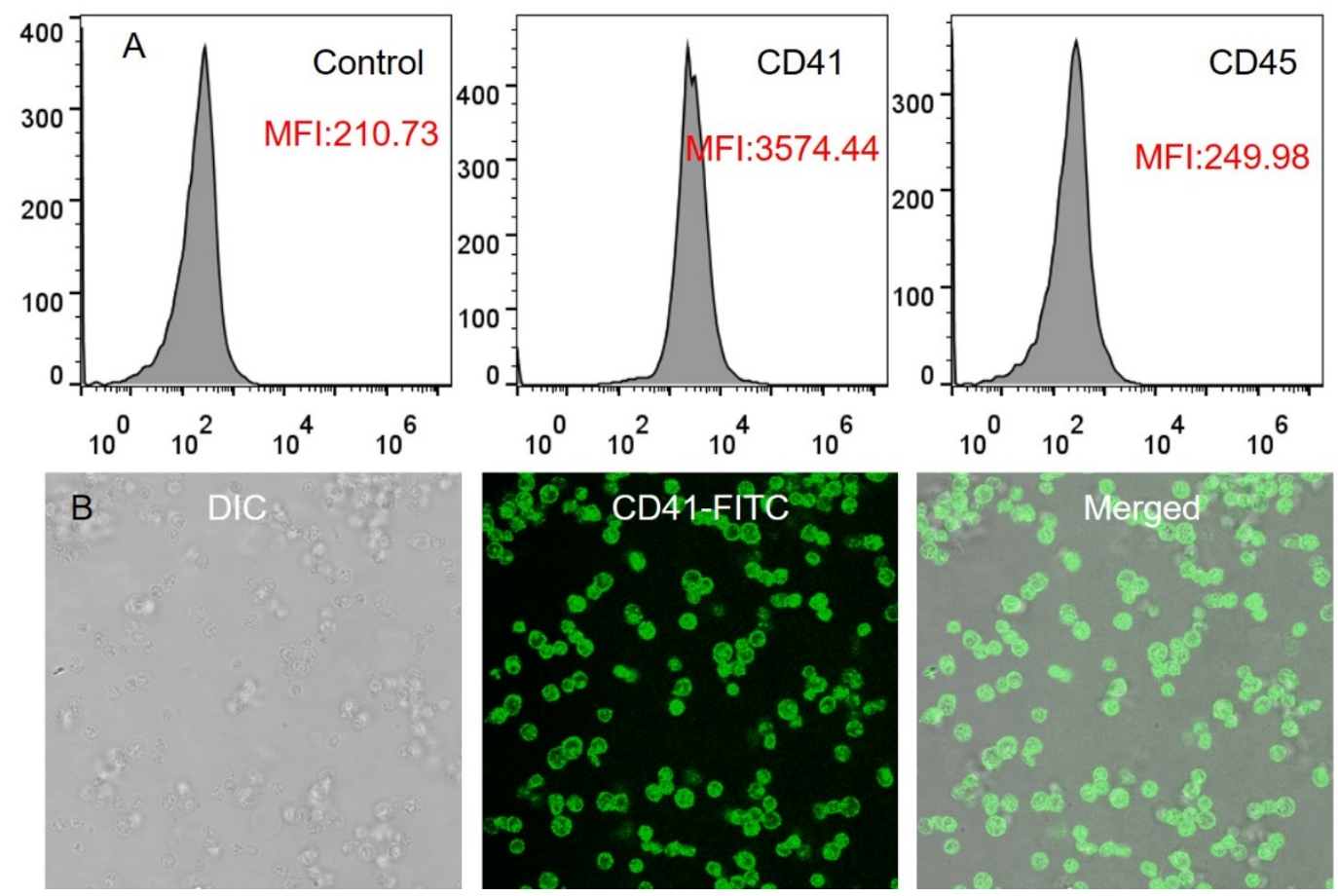

Figure S5. (A) FACS study of purified platelets that were doubly stained with FITCconjugated, anti-CD45 (white blood cell marker) and anti-CD41 (platelet marker). (B) Bright and fluorescence CLSM images of the collected platelet labeled with CD41FITC.

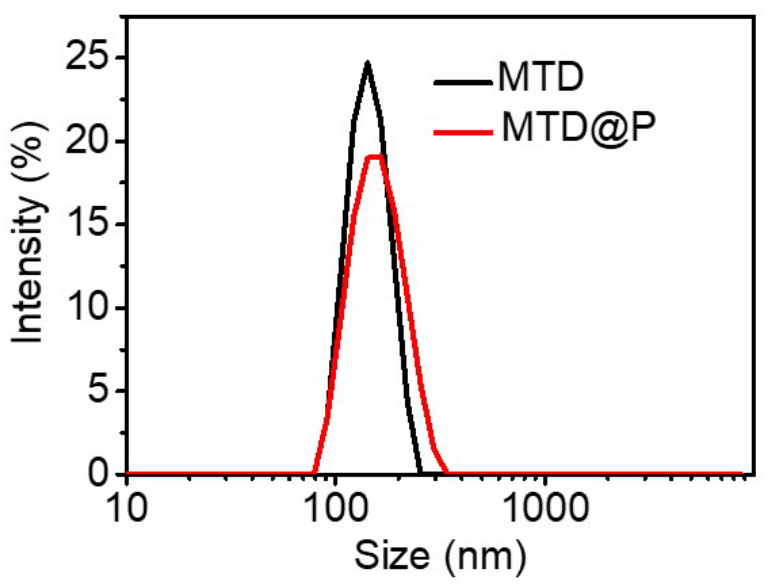

Figure S6. Hydrodynamic diameters of MTD and MTD@P nanoparticles. 


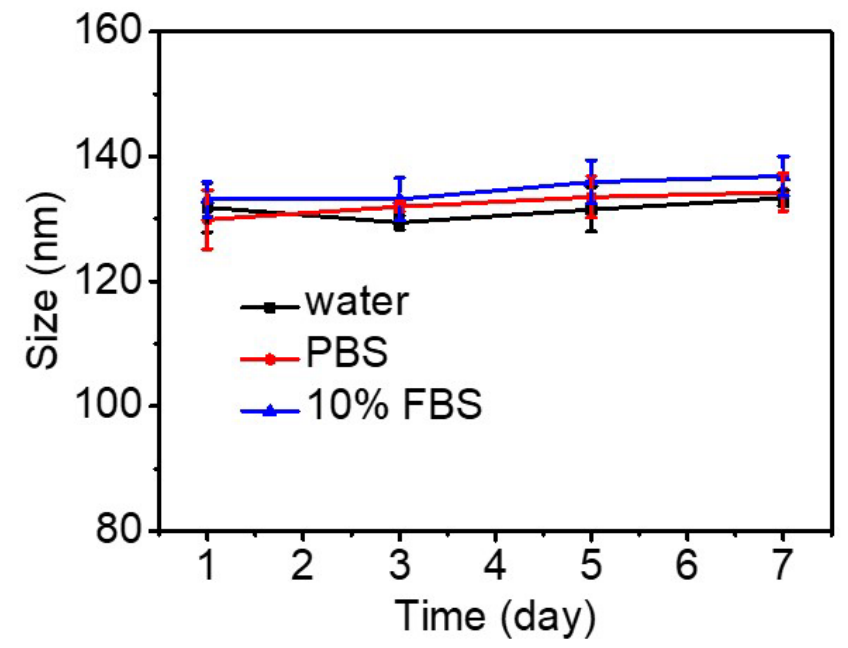

Figure S7. The stability test of MTD@P nanoparticles in different medium for 7 days.
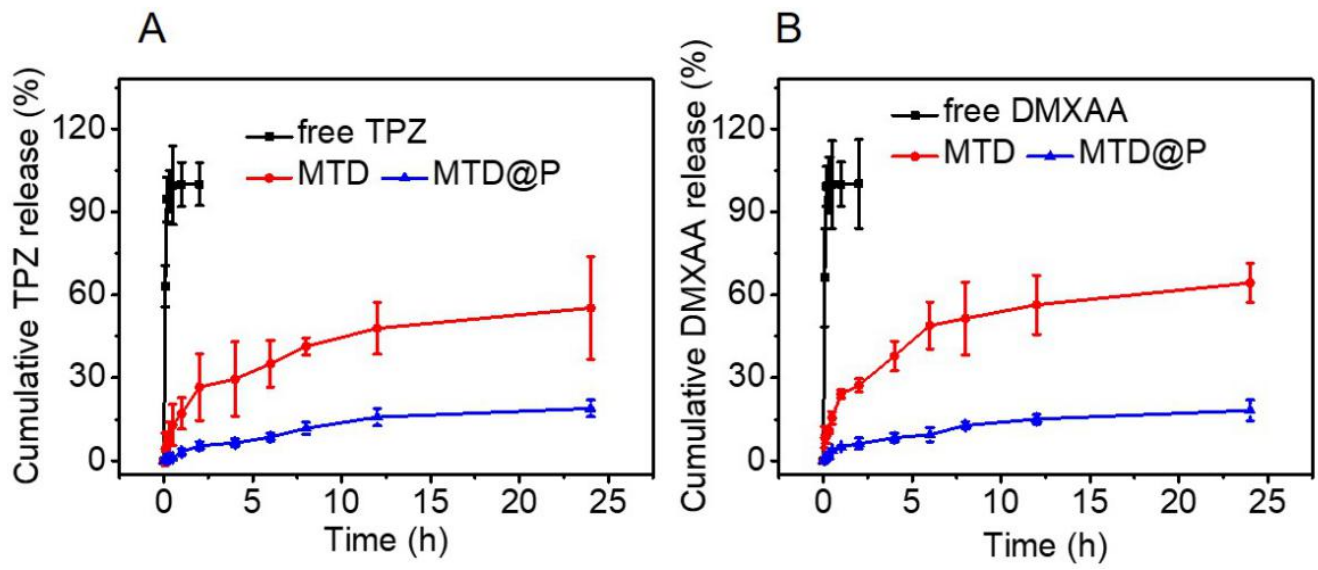

Figure S8. The in vitro release profile of TPZ (A) and DMXAA (B) from free TPZ or DMXAA, MTD and MTD@P atpH 7.4. 


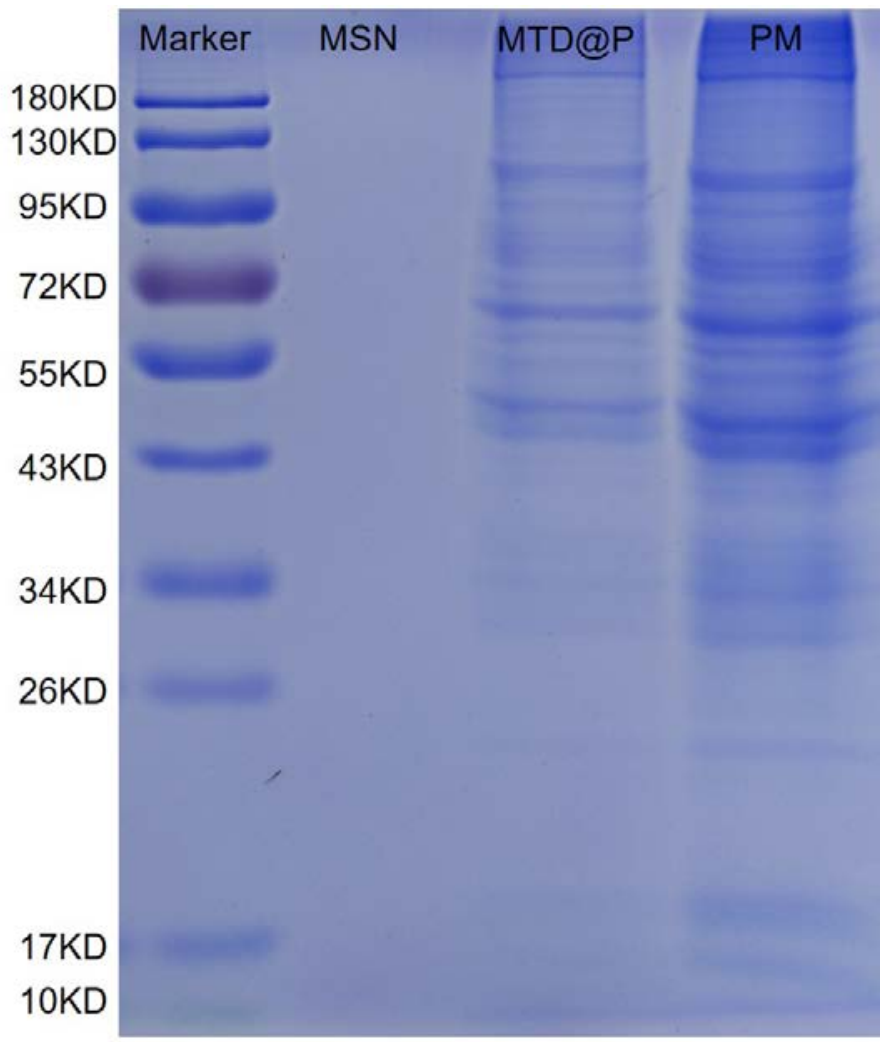

Figure S9. SDS-PAGE protein analysis of MSN, MTD@P and PM.

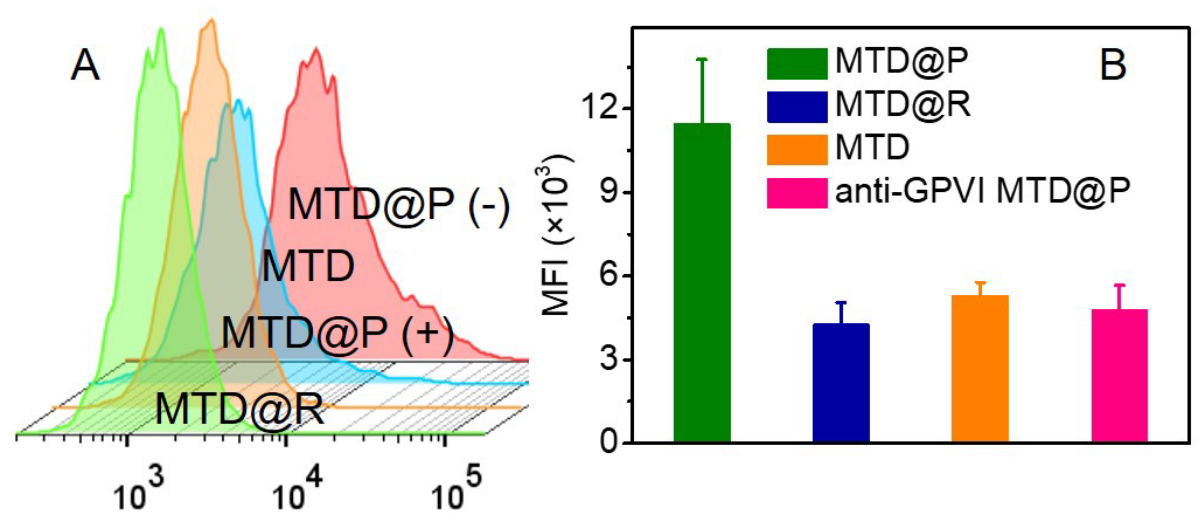

Figure S10. (A) Quantitative analysis toward the fluorescence intensity in MAEC cells after 4 h co-incubation with 100 g/mL of MTD@P, MTD@R, MTD and MTD@P (+ represented MTD@P was pre-treated with GPVI antibody) (B) Mean fluorescence intensity in MAEC cells after different treatments. 

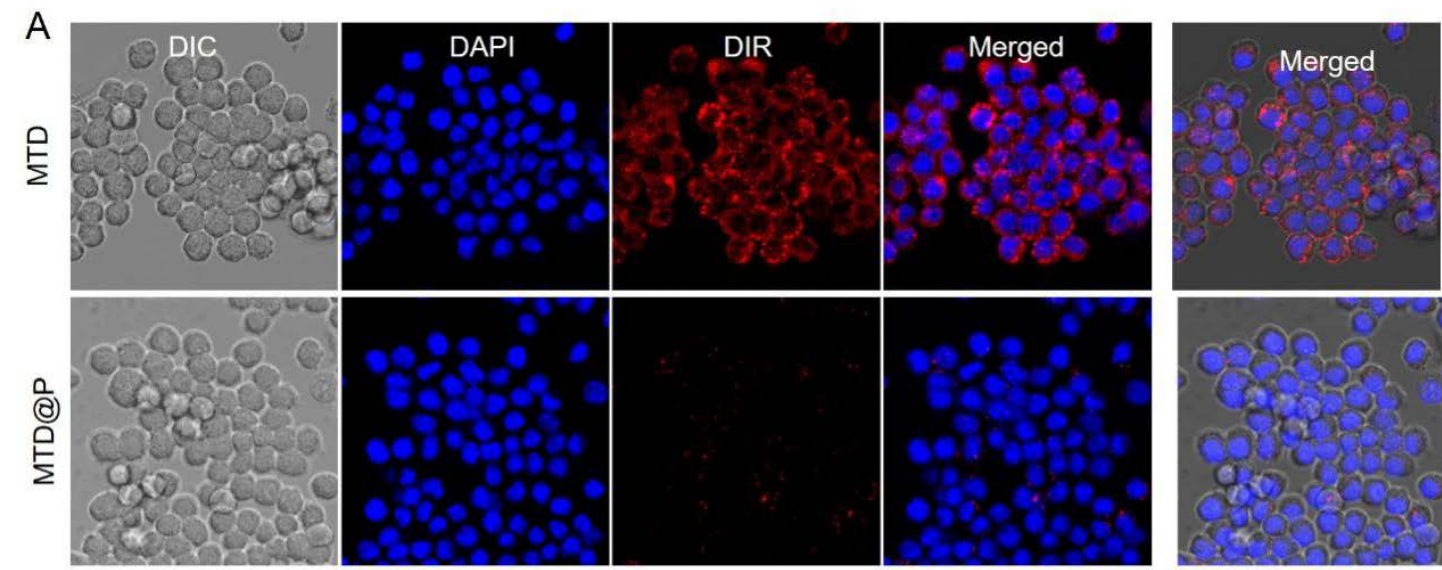

B

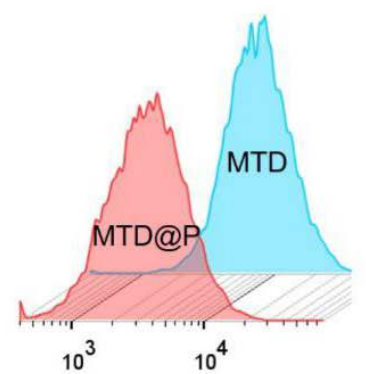

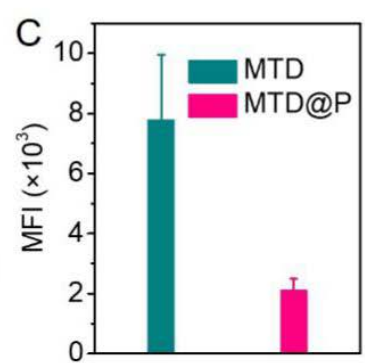

Figure S11. (A) Fluorescence images of RAW264.7 cells after the treatments with MTD and MTD@P, respectively. (B) Quantitative analysis toward the fluorescence intensity in RAW264.7 cells after 2 h co-incubation with MTD and MTD@P. (C) Mean fluorescence intensity of DIR labeled nanoparticles in RAW264.7 cells after different treatments.

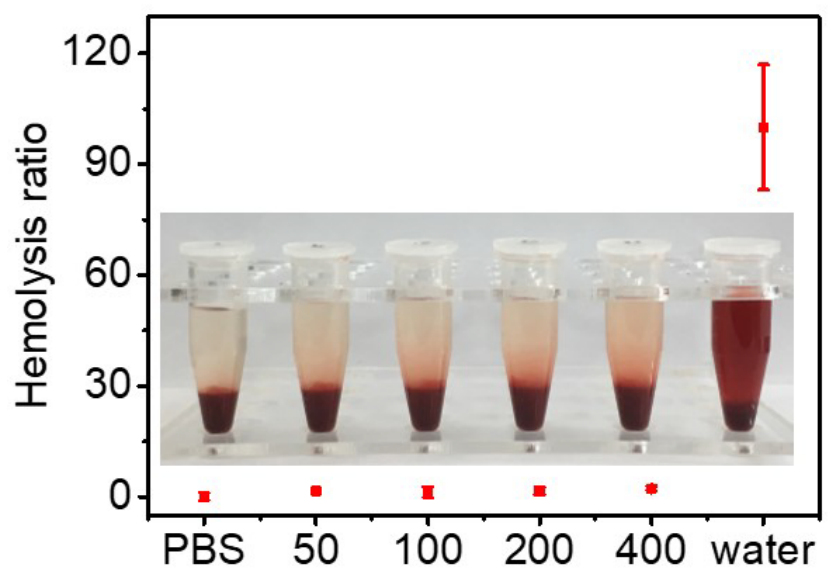

Figure S12. Hemolysis test of MTD@P at different concentrations (0, 50, 100, 200, $400 \mu \mathrm{g} / \mathrm{mL}$ ) in PBS. 


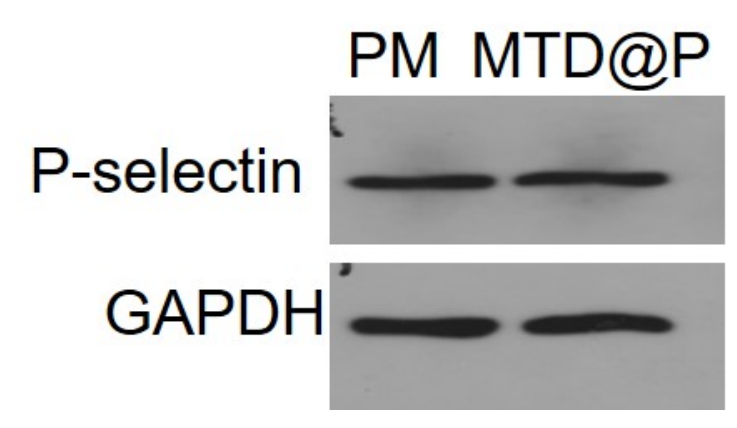

Figure S13. Western blotting analysis for P-Selectin expression on PM and MTD@P.

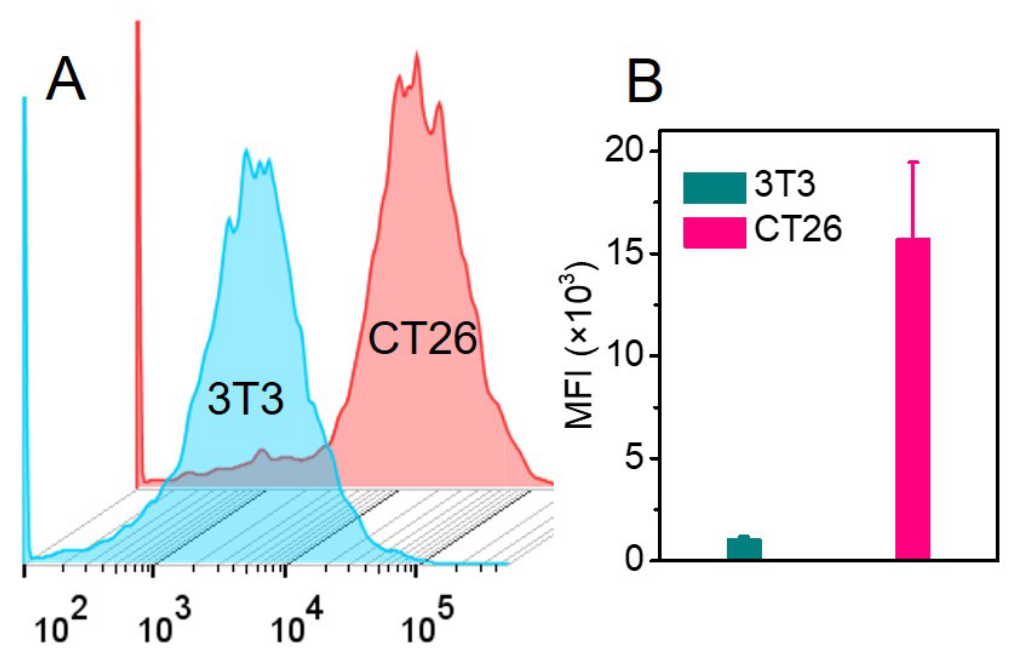

Figure S14. (A) Quantitative analysis toward the fluorescence intensity in 3T3 and CT26 cell after 2 h co-incubation with $100 \mu \mathrm{g} / \mathrm{mL}$ of MTD@P. (B) Mean fluorescence intensity in 3T3 and CT26 cell after co-incubation with MTD@P. 


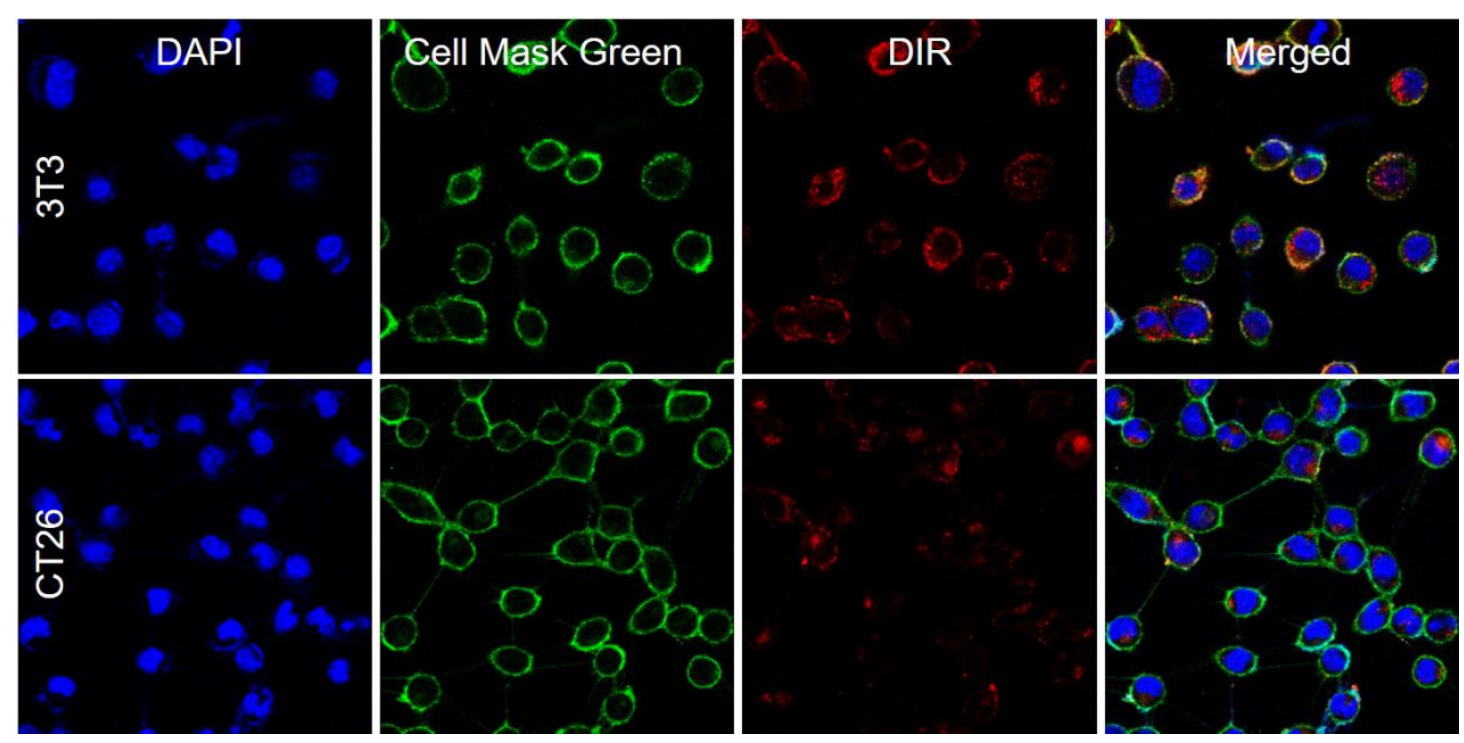

Figure S15. Fluorescence images of 3T3 (upper) and CT26 (lower) cells after 2 h coincubation with $100 \mu \mathrm{g} / \mathrm{mL}$ of MTD.
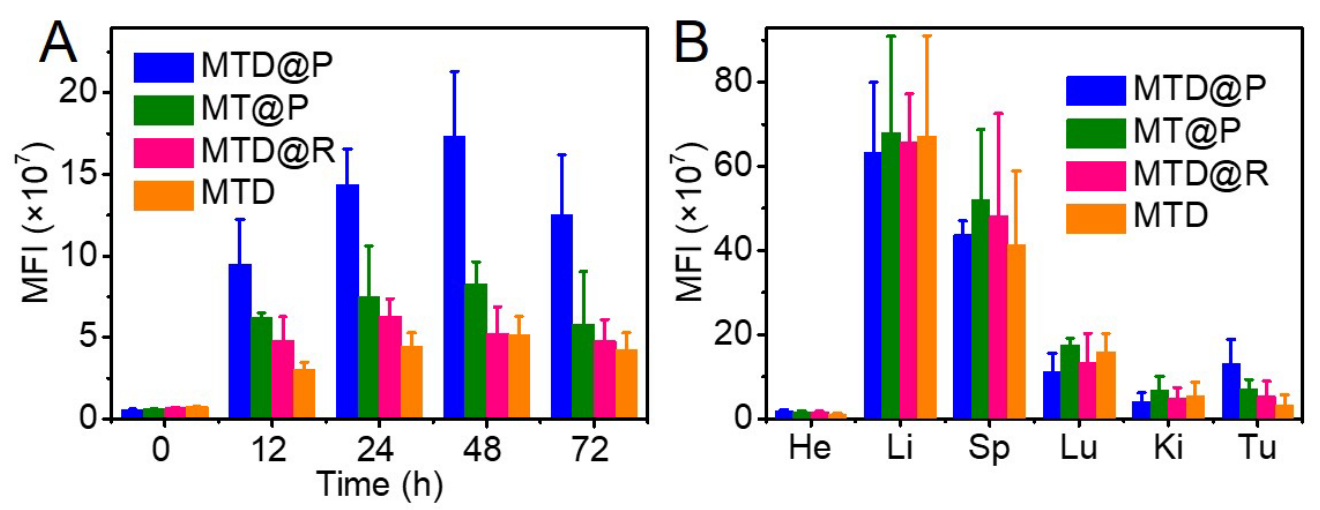

Figure S16. (A) Variation of mean fluorescence intensity at tumor sites in the mice with time after the treatments with MTD@P, MT@P, MTD@R and MTD. (B) Mean fluorescence intensity of major organs in the mice treated with MTD@P, MT@P, MTD@R and MTD. (He: Heart; Li: Liver; Sp: Spleen; Lu: Lung; Ki: Kidney; Tu: Tumor). The organs were obtained at $72 \mathrm{~h}$ post injection. 

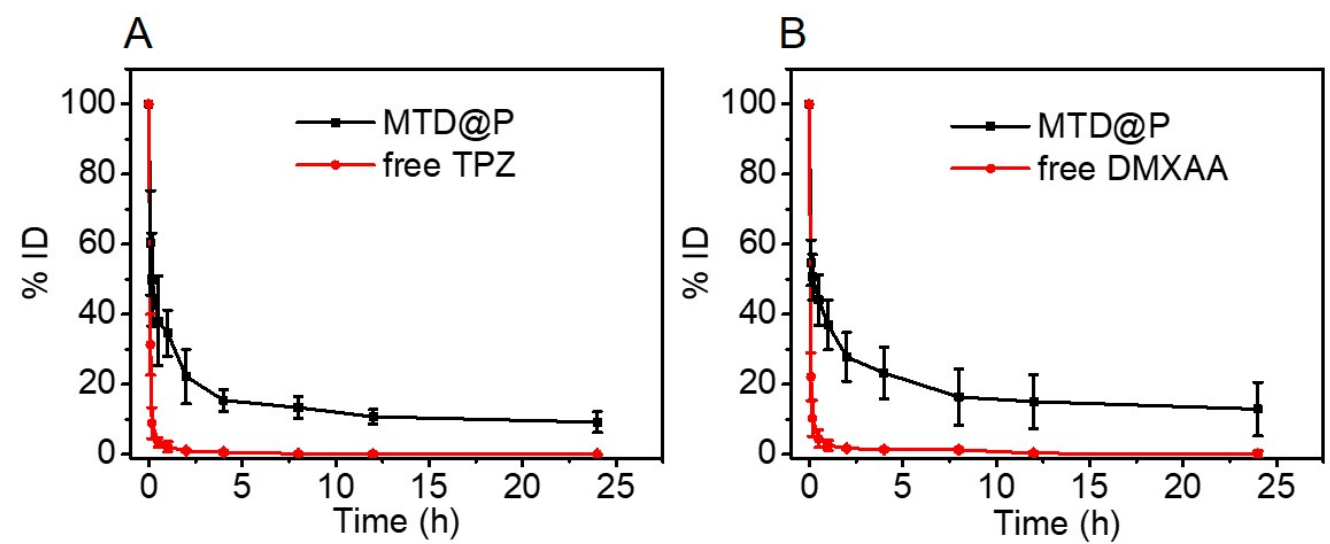

Figure S17. In vivo pharmacokinetic curves of TPZ (A) and DMXAA (B) after intravenous injected with free TPZ or DMXAA and MTD@P, respectively.

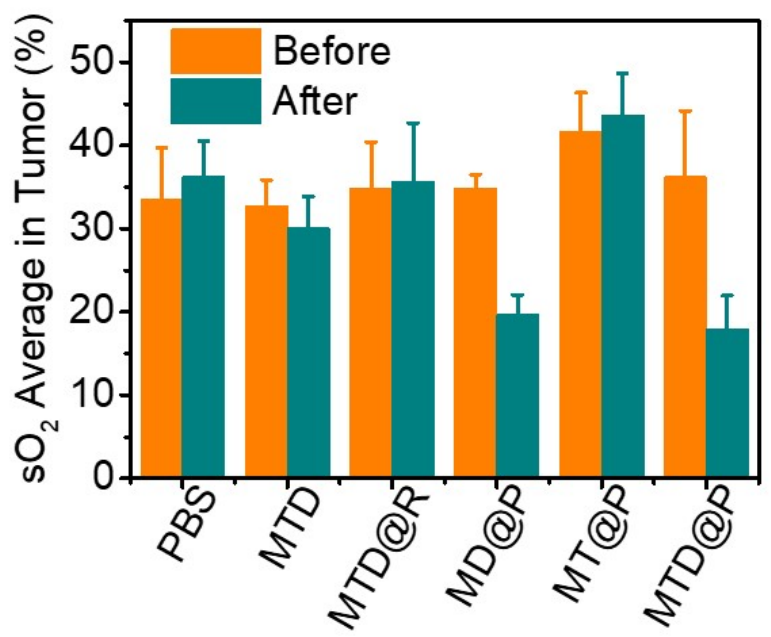

Figure S18. Variation of intratumoral average $\mathrm{sO}_{2}$ in different groups obtained before (0 h) and after (4 h) the administrations with different nanoparticles. 


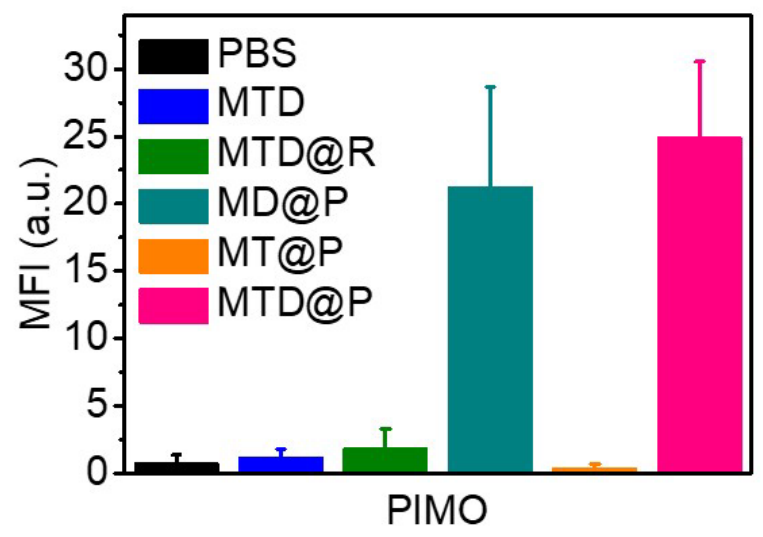

Figure S19. Mean fluorescence intensity in immunofluorescence images of the tumor slices stained with of pimonidazole antibody after different treatments.

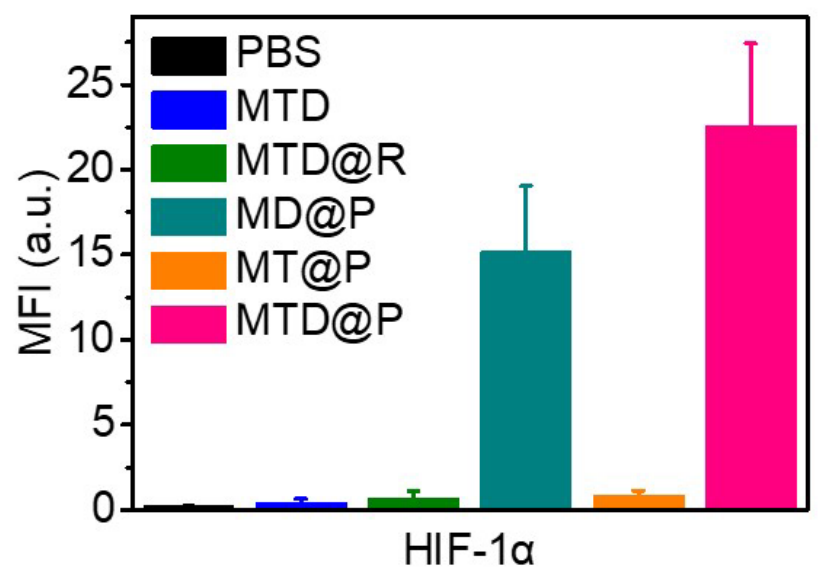

Figure S20. Mean fluorescence intensity in immunofluorescence images of the tumor slices stained with of HIF-1 $\alpha$ antibody after different treatments. 

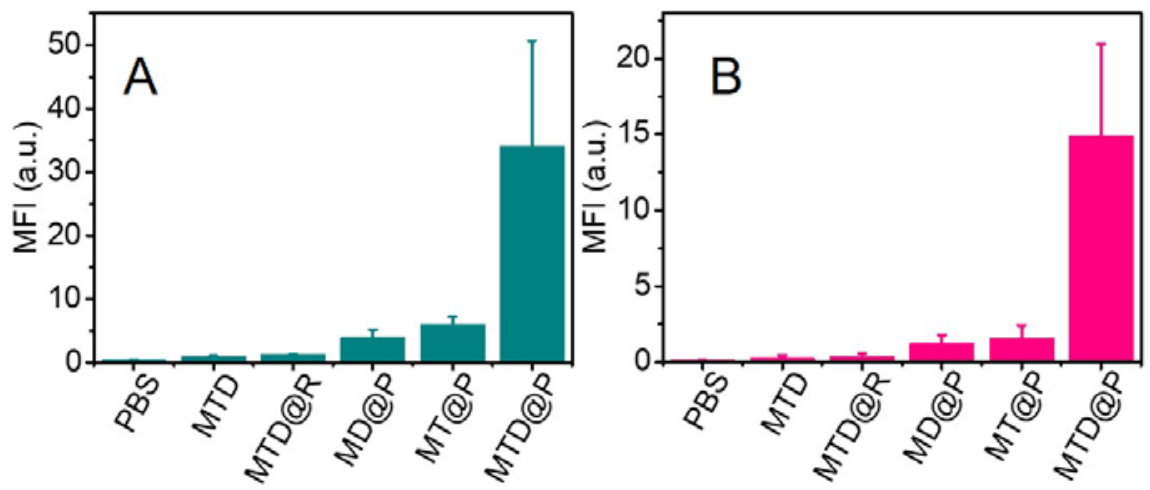

Figure S21. Mean fluorescence intensity of TUNEL (A) and caspase-3 (B) antibody staining images of the tumor slices after different treatments

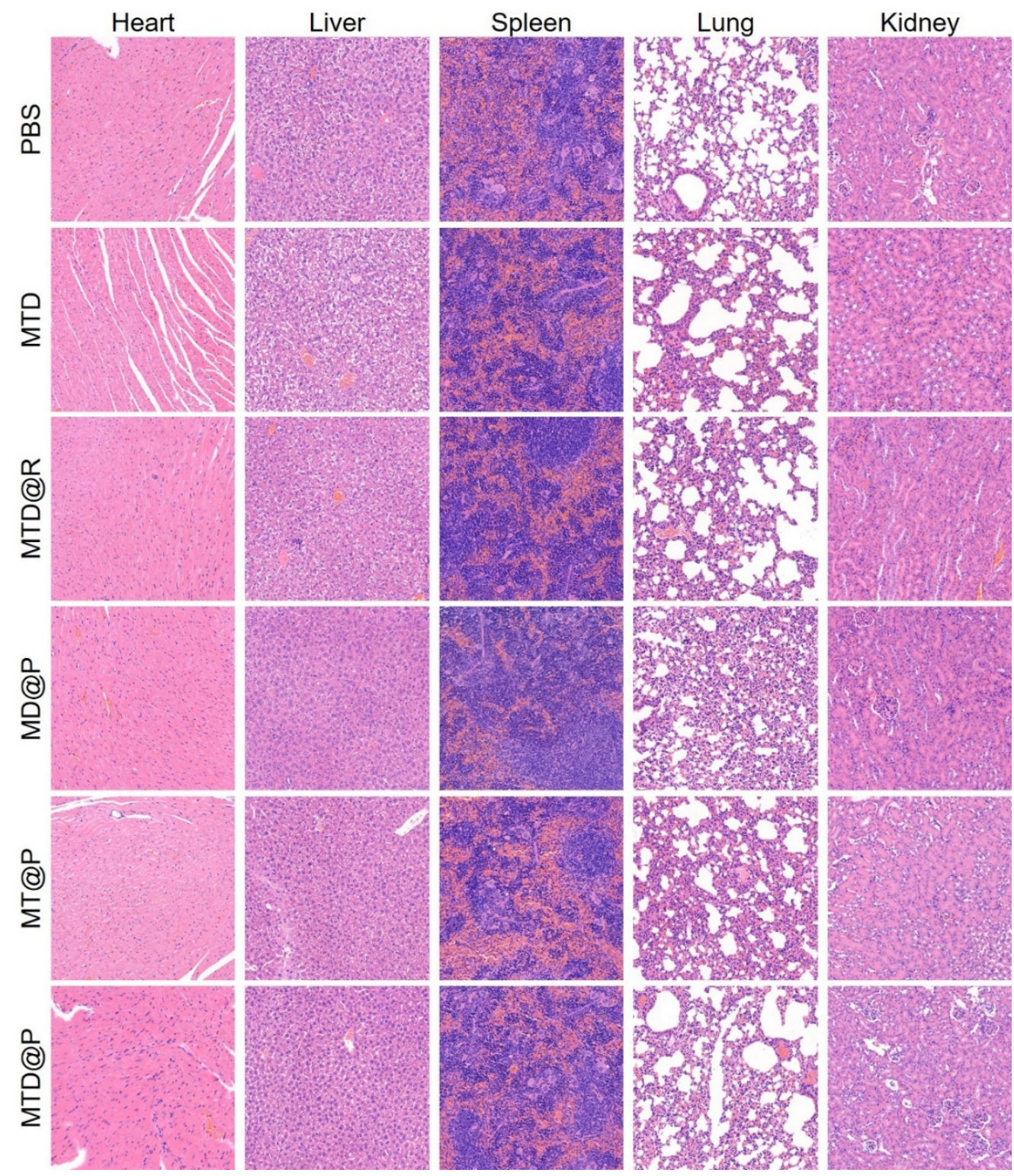

Figure S22. H\&E staining images of major organ slices after different treatments. 

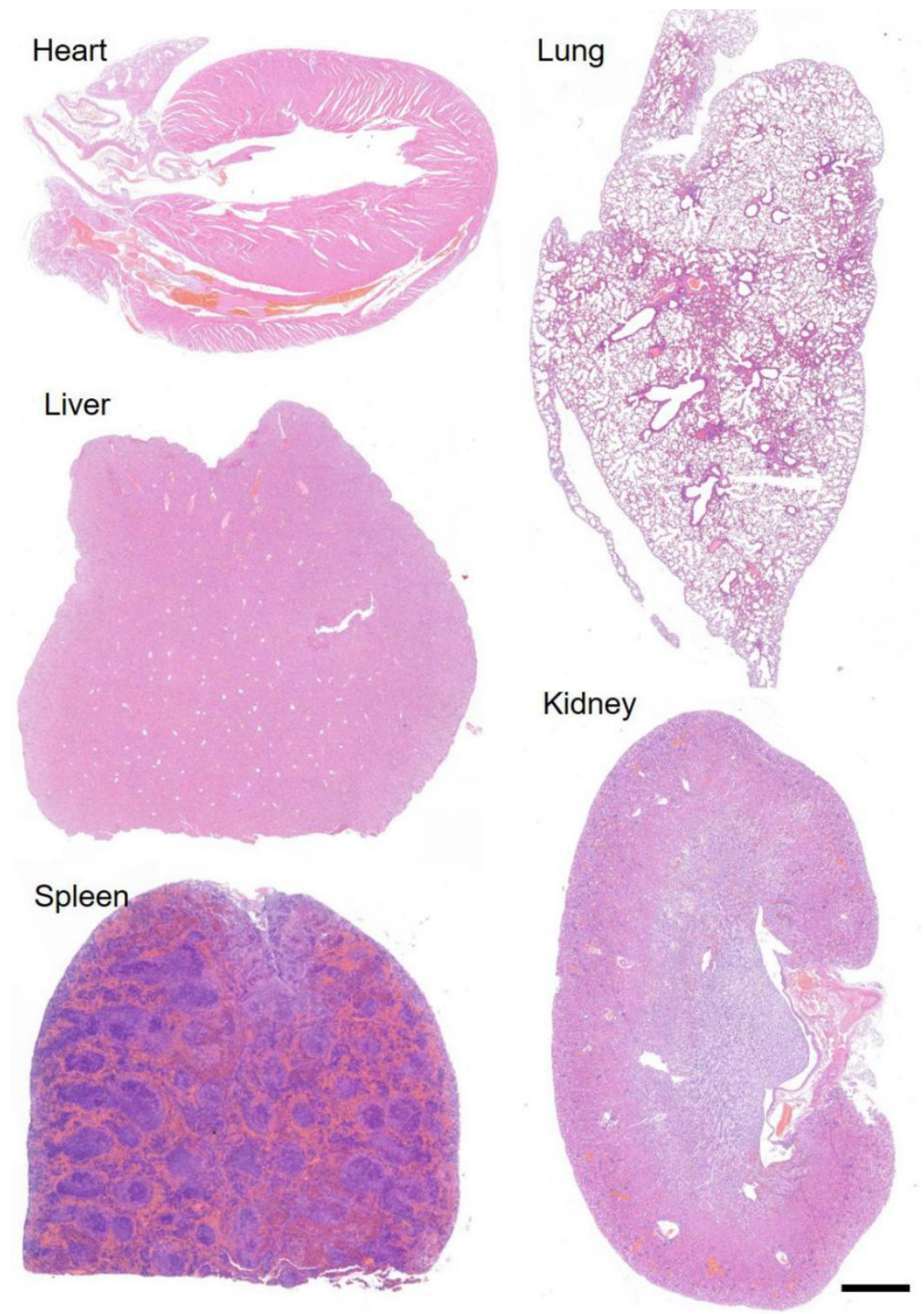

Figure S23. Full scan H\&E staining images of major organ slices of healthy mice. Scale bar: $1 \mathrm{~mm}$. To demonstrate the specificity of DMXAA to tumor vasculature, free DMXAA was intravenously injected into healthy mice and CT26 tumor-bearing mice (shown in Figure S24), respectively. After 4 hours, the mice were sacrificed and the main organs and tumors were collected for H\&E staining by microscopy. There were no obvious abnormalities in the normal tissues of healthy mice and tumor-bearing mice, but a lot of bleeding spots appeared in tumors, strongly confirming the high specificity of DMXAA to tumor vasculature. 


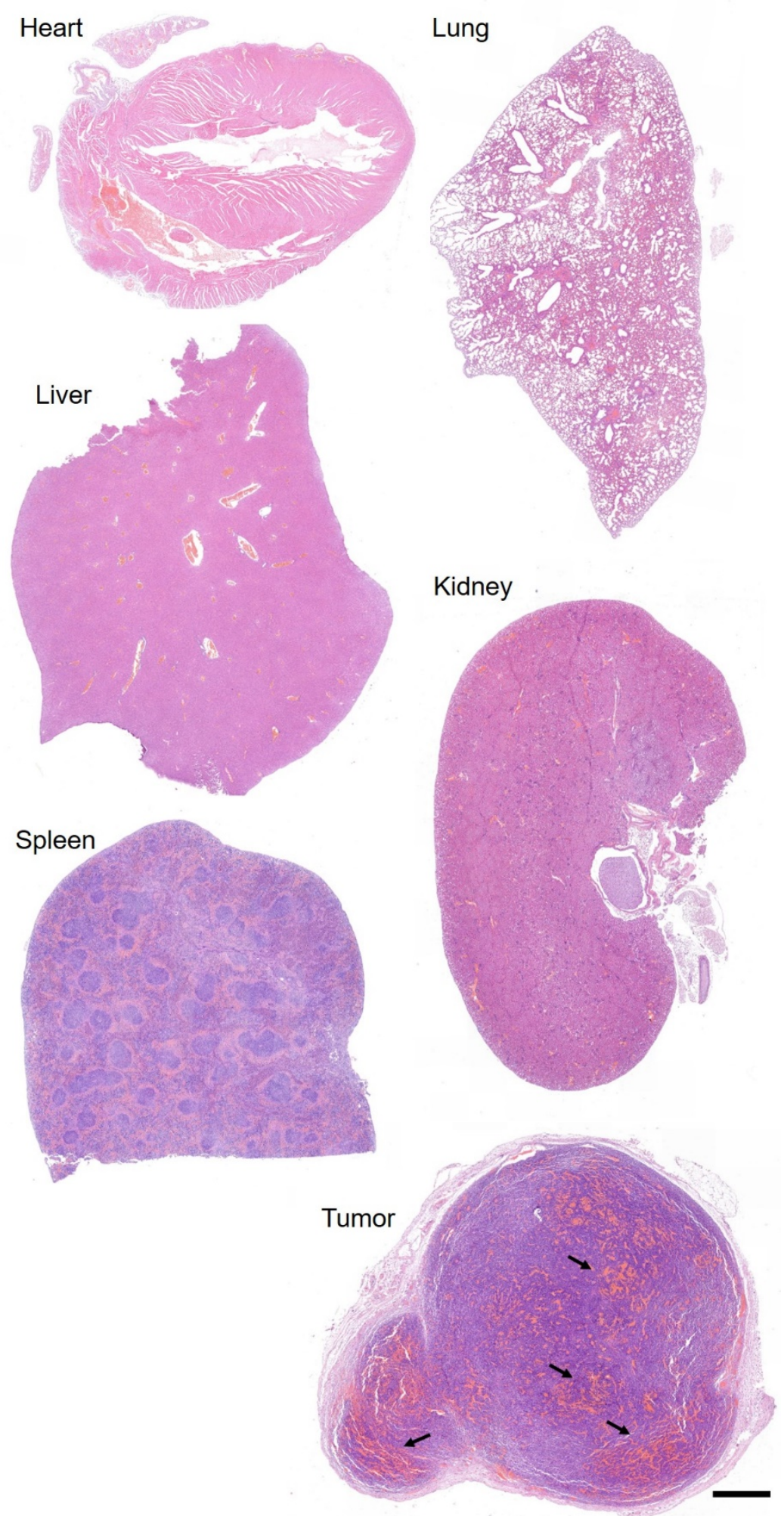

Figure S24. Full scan H\&E staining images of major organ slices of CT26 tumorbearing mice. Scale bar: $1 \mathrm{~mm}$. Bleeding spots are indicated by black arrows. 
Table S1. Blood routine analysis post different treatments (Major items: RBC: red blood cell, WBC: white blood cell, PLT: platelets, HCT: hematocrit, MCV: mean corpuscular volume, HGB: hemoglobin).

\begin{tabular}{|c|c|c|c|c|c|c|}
\hline & PBS & MTD & MTD@R & MD@P & MT@P & MTD@P \\
\hline RBC $\left(10^{\wedge} 12 / \mathrm{L}\right)$ & $5.51 \pm 2.20$ & $7.57 \pm 0.32$ & $7.46 \pm 0.61$ & $6.36 \pm 0.67$ & $7.15 \pm 0.49$ & $7.26 \pm 0.51$ \\
\hline WBC (10^9/L) & $7.10 \pm 1.90$ & $9.90 \pm 1.45$ & $9.60 \pm 1.76$ & $5.60 \pm 2.69$ & $11.27 \pm 2.06$ & $8.73 \pm 1.66$ \\
\hline PLT (10^9/L) & $487.67 \pm 84.18$ & $602 \pm 103.70$ & $679.67 \pm 119.88$ & $796.33 \pm 114.22$ & $602.67 \pm 48.27$ & $777.67 \pm 69.43$ \\
\hline HCT (\%) & $30.37 \pm 7.60$ & $39.00 \pm 1.25$ & $38.83 \pm 3.31$ & $33.43 \pm 3.85$ & $36.63 \pm 2.77$ & $37.07 \pm 2.14$ \\
\hline MCV (fL) & $58.43 \pm 12.46$ & $51.53 \pm 0.86$ & $52.03 \pm 0.68$ & $52.57 \pm 1.15$ & $51.20 \pm 0.53$ & $51.07 \pm 1.01$ \\
\hline HGB (g/L) & $111.33 \pm 15.70$ & $112.00 \pm 3.46$ & $115.00 \pm 8.54$ & $100.67 \pm 5.51$ & $108.33 \pm 6.66$ & $108.67 \pm 4.16$ \\
\hline
\end{tabular}

Table S2. Blood biochemistry analysis post different treatments. (TP: total protein, ALB: albumin, GLO: globulin, TBIL: total bilirubin, ALT: alanine aminotransferase, AST: aspartate aminotransferase, GGT: $\gamma$-glutamyl transpeptidase, UREA: urea, GLU: glucose).

\begin{tabular}{|c|c|c|c|c|c|c|}
\hline & PBS & MTD & MTD@R & MD@P & MT@P & MTD@P \\
\hline TP (g/L) & $29.60 \pm 0.50$ & $35.37 \pm 5.34$ & $30.80 \pm 5.37$ & $29.73 \pm 2.44$ & $29.93 \pm 0.58$ & $30.55 \pm 0.49$ \\
\hline ALB (g/L) & $22.80 \pm 1.14$ & $22.87 \pm 0.06$ & $22.67 \pm 0.65$ & $23.10 \pm 1.11$ & $22.07 \pm 0.31$ & $23.07 \pm 0.38$ \\
\hline GLO (g/L) & $6.80 \pm 0.00$ & $15.50 \pm 1.84$ & $13.50 \pm 0.00$ & $8.30 \pm 0.00$ & $8.80 \pm 0.00$ & $7.40 \pm 0.00$ \\
\hline TBIL ( $\mu$ mol/L) & $2.50 \pm 1.35$ & $0.57 \pm 0.38$ & $1.40 \pm 0.82$ & $1.73 \pm 0.64$ & $2.47 \pm 0.25$ & $2.77 \pm 0.25$ \\
\hline ALT (U/L) & $22.67 \pm 4.04$ & $25.33 \pm 1.15$ & $21.33 \pm 1.15$ & $21.67 \pm 3.06$ & $23.33 \pm 2.52$ & $25.00 \pm 2.00$ \\
\hline AST (U/L) & $69.67 \pm 6.03$ & $114.00 \pm 52.05$ & $168.33 \pm 41.00$ & $150.33 \pm 29.14$ & $92.00 \pm 43.86$ & $106.33 \pm 45.98$ \\
\hline GGT (U/L) & $2.93 \pm 0.75$ & $3.27 \pm 2.16$ & $3.60 \pm 0.40$ & $4.00 \pm 1.57$ & $3.83 \pm 0.51$ & $2.83 \pm 0.06$ \\
\hline $\begin{array}{c}\text { UREA } \\
(\mathrm{mmol} / \mathrm{L})\end{array}$ & $3.90 \pm 0.72$ & $5.59 \pm 0.62$ & $4.75 \pm 1.05$ & $5.26 \pm 1.30$ & $5.36 \pm 0.54$ & $5.64 \pm 1.32$ \\
\hline GLU (mmol/L) & $8.98 \pm 0.50$ & $8.32 \pm 2.30$ & $9.23 \pm 0.68$ & $8.43 \pm 1.47$ & $8.00 \pm 0.77$ & $8.12 \pm 1.00$ \\
\hline
\end{tabular}

